
échanges littéraires et culturels (XIVe-XVIe siècle)

\title{
L'Europe et ses « Européens » vus par Enea Silvio Piccolomini (1458)
}

L'Europa ed i suoi «Europei» visti da Enea Silvio Piccolomini (1458)

Europe and Its "Europeans" Seen by Enea Silvio Piccolomini (1458)

\section{Serge Stolf}

\section{OpenEdition}

\section{Journals}

Édition électronique

URL : http://journals.openedition.org/cei/5225

DOI : $10.4000 /$ cei.5225

ISSN : 2260-779X

\section{Éditeur}

UGA Éditions/Université Grenoble Alpes

Édition imprimée

ISBN : 978-2-37747-063-7

ISSN : $1770-9571$

Référence électronique

Serge Stolf, «L'Europe et ses « Européens » vus par Enea Silvio Piccolomini (1458) », Cahiers d'études italiennes [En ligne], 27 | 2018, mis en ligne le 30 septembre 2018, consulté le 26 mars 2021. URL: http://journals.openedition.org/cei/5225 ; DOI : https://doi.org/10.4000/cei.5225

Ce document a été généré automatiquement le 26 mars 2021

(C) ELLUG 


\title{
L’Europe et ses « Européens » vus par Enea Silvio Piccolomini (1458)
}

\author{
L'Europa ed i suoi «Europei» visti da Enea Silvio Piccolomini (1458) \\ Europe and Its "Europeans" Seen by Enea Silvio Piccolomini (1458)
}

\section{Serge Stolf}

1 C'est un historien italien du xx siècle, Federico Chabob, qui, dès 1947, s'est intéressé parmi les premiers à l'histoire de l'idée d'Europe. Ses réflexions ont abouti à une remarquable synthèse, parue en 1961, où il reprenait, depuis ses origines, le chemin, fait de continuités et de ruptures, suivi par cette idée. Le texte remontait à l'immédiat après-guerre, quand se posait l'urgente question politique, et donc culturelle, de la survie d'une Europe et de sa civilisation. Il déclarait : «De fait, c'est la foi en quelques valeurs suprêmes, spirituelles et morales, élaborées par notre civilisation européenne, qui a fait naître le désir de remonter historiquement l'iter de cette civilisation ${ }^{1}$. » Dans les limites d'un court article, je me propose de marquer l'une des étapes de cette histoire avec Enea Silvio Piccolomini et ses considérations sur l'Europe, exprimées dans divers écrits, qu'il condensera dans le De Europa, écrit entre février et août 1458, peu avant son élection au pontificat qu'il assuma sous le nom de Pie II. L'ouvrage, rédigé en latin, mérite sans doute plus que des citations cursives dans les travaux d'histoire générale de l'Europe. Alors que la réappropriation littéraire du mythe d'Európe est manifeste à la fin du $\mathrm{xv}^{\mathrm{e}}$ siècle - «"Europa”, suona il lito, "Europa, riedi” " (" "Europe”, fait écho le rivage, "Europe, reviens" »), crient ses compagnes dans les Stanze du Politien $^{2}-$, c'est aussi une forme de réappropriation de l'Europe, envisagée comme réalité tout à la fois géographique, politique et spirituelle, qu'entreprend Piccolomini dans son ouvrage ${ }^{3}$. Sa rédaction intervient à un moment significatif, celui où la perception d'une menace pesant sur l'identité de cet espace humain et politique ${ }^{4}-$ un territoire -, et culturel, naît des progrès qu'y font les avancées militaires ottomanes. Les questions soulevées par ce texte sont multiples : en même temps qu'il peut être considéré dans sa dimension polémique, à savoir le sursaut d'une Europe sur la défensive, il interroge sur l'idée que se fait de l'Europe et des "Européens » un humaniste qui en est depuis un quart de siècle l'observateur attentif. Héritier d'une 
tradition classique et d'une culture médiévale qui avant lui avaient pensé l'Europe ${ }^{5}$, il perçoit avec acuité les divers aspects d'une crise dont la prise de Constantinople, la "seconde Rome ", le 29 mai 1453, par les Turcs de Mahomet II, a été l'épisode le plus marquant pour la conscience d'un homme comme Piccolomini, et pour celle d'autres contemporains ${ }^{6}$. Cet événement a créé une situation nouvelle, ou inédite, qui explique l'urgence de penser le plus clairement possible la réalité européenne du moment et d'en tirer, non seulement un instantané, mais aussi une idée fondatrice et durable ${ }^{7}$.

2 L'Europe médiévale "comme réalité et comme représentation", comme l'écrivait Jacques Le Goff, qui ajoutait : «Seul le pape Pie II [...] a eu une idée claire de l'Europe ${ }^{8}$.» Il reste à comprendre comment cette idée a surgi dans sa clarté, dans quelle continuité elle s'est inscrite, au terme de quelles ruptures elle s'est construite. Le De Europa n'a rien d'une vision abstraite. Il y est question d'hommes, de peuples, de nations, de migrations, de mœurs et de coutumes, de considérations qui tiennent à l'ethnographie, à la géographie, à l'histoire, à une réalité humaine qui n'a rien d'immobile, et pourtant se caractérise et se « découvre » européenne. Piccolomini ne donne-t-il pas une image éloignée de la réalité d'hommes qui, sous un même continent, sont plus attachés à leur diversité, à ce qui les sépare, qu'aux traits communs ou aux affinités qui les rapprochent? Cette représentation de l'Europe participe-t-elle d'une projection intellectualisée ou d'une nécessité pragmatique, essentiellement politique, de donner de l'Europe et de ses habitants une image cohérente et surtout, cohésive? De plus, le De Europa de Piccolomini présente l'intérêt d'être le point de vue d'un Italien, qui a certes vécu autant hors d'Italie que sur son sol natal (Sienne et la Toscane), et a beaucoup voyagé en Europe, mais dont la formation intellectuelle s'est faite en Italie, dans les centres d'enseignement humaniste de la péninsule ${ }^{9}$. Existerait-il alors, sur le plan culturel, une forme d'italo-centrisme? Pour aborder ces divers points, le plus brièvement possible, j'examinerai d'abord l'hypothèse d'un passage ou d'un transfert de la Chrétienté à l'Europe, s'agissant de coïncidences plus apparentes qu'évidentes. Dans un deuxième temps, je m'attacherai à la question des «Européens » - substantif qui apparaît pour la première fois dans le De Europa - et de l'articulation entre communauté (universitas) et diversité d'appartenance à l'Europe. Un dernier point essaiera de déterminer dans quelles mesure et limites l'Europe vue par Piccolomini reflète un point de vue « italien ».

\section{De la christianitas à l'Europa?}

3 Le nom Európe est désigné pour la première fois comme continent, dans un Hymne homérique du vi $\mathrm{I}^{\mathrm{e}}$ siècle av. J.-C. ${ }^{10}$. C'est par opposition à l'Asie et à l'Afrique que l'Europe se constitue, chez les géographes grecs, comme entité géographique, et que les limites la séparant de l'Asie sont à peu près fixées par le fleuve Tanaïs (le Don actuel), et par le détroit du Bosphore et celui des Dardanelles (l'Hellespont des Anciens). Piccolomini, lecteur de la Géographie de Strabon, dont la traduction manuscrite de Guarino Veronese et Gregorio Tifernate ${ }^{11}$ circule à partir de 1456, conserve dans le De Europa, conçu comme une description allant de l'est vers l'ouest, cette frontière orientale du Tanaïs et la séparation des deux continents ( « c'est le seul espace séparant l'Europe de l'Asie ; les Anciens l'ont appelé Bosphore de Thrace $\left.{ }^{12} »\right)$, mais fait considérablement reculer, au nord, les terrce incognitee des Grecs et des Romains : c'est ce même découpage spatial qu'on trouve, un siècle plus tard, dans la carte de Mercator (1589) ou d'Ortelius, dans 
son atlas de 1575. Le De Europa illustre ce rapport essentiel entre territoire ${ }^{13}$ et histoire, dont le caractère principal est de refléter une réalité mouvante. Lorsque un poème, attribué à Angilbert, daté de 799, qualifie Charlemagne de "Europce venerandus apex, pater optimus ${ }^{14}$ » («cime vénérable et père très bon de l'Europe »), cette Europe, écrit J. Le Goff, « ne comprend pas les îles Britanniques [...], la péninsule Ibérique, conquise pour l'essentiel par les musulmans, l'Italie du Sud et la Sicile, également aux mains des Sarrasins ; ni, enfin, la Scandinavie, restée païenne ${ }^{15}$ » : en somme, « une Europe avant tout germanique ${ }^{16}$ ». Constantinople même se voit reléguée "aux confins de l'Europe ${ }^{17} »$. En des termes presque identiques, Dante, au chant VI (v. 5) situe Byzance "à l'extrémité de l'Europe » («ne lo stremo d'Europa »).

La christianitas se définit aussi par opposition aux «barbares » que sont les païens et les «infidèles", et ne s'identifie pas à l'Europe, mais aux peuples qui composent cette communauté, universitas christianorum, en et hors d'Europe. La Chrétienté - menacée en Asie Mineure et en Palestine - l'est aussi dans la seule partie qu'elle occupe en Europe, explique le pape Urbain II au Concile de Clermont (1095) : « Ainsi donc, Turcs et Sarrasins font la guerre à cette petite partie de notre monde ${ }^{18}$.» La Chrétienté va progressivement s'identifier aussi à l'orbis christianus, ensemble des peuples et royaumes, terme qu'utilisera encore Piccolomini : « [Rome] est désormais en un coin du monde chrétien ${ }^{19}$.» Il faut attendre le début du xiII ${ }^{\mathrm{e}}$ siècle pour que, par la christianisation progressive des peuples venus du nord et de l'est - Scandinaves et Hongrois - la Chrétienté englobe toute l'Europe et que celle-ci soit vue comme totalement chrétienne ${ }^{20}$.

5 En avril 1432, Piccolomini arrive à Bâle où se tient le concile. Il y demeurera jusqu'en novembre 1442, date à laquelle il entrera au service de Frédéric III de Habsbourg, empereur nominal, et y défendra les intérêts impérialistes. Pendant cette période où s'installe le conflit d'autorité entre Bâle et le pape Eugène IV, la correspondance et les discours de Piccolomini attestent que l'identification de l'Europe à la Chrétienté acquiert une acception tantôt religieuse, tantôt politique. Le lieu du concile, Bâle, est défini comme "centre de la Chrétienté ou proche de ce centre ${ }^{21}$ ». Dans un discours prononcé devant le duc de Milan en 1436, Piccolomini déclare : « [les Turcs] ont étendu leur domination d'Asie en Europe [...] et, pour les chasser de la Grèce, il n'y faudrait pas un seul état ou une seule puissance, mais la Chrétienté tout entière ${ }^{22}$. »

La menace qui, deux siècles et demi plus tôt, justifiait la reconquête des territoires perdus de cette Chrétienté en Asie, a fait place à l'occupation, en Europe, de terres grecques chrétiennes. C'est donc bien l'Europe, avec son dernier bastion byzantin, qui doit s'unir pour en chasser les Ottomans. Piccolomini, devenu le soutien d'un absolutisme impérial, voit en Frédéric III le souverain capable d'assurer à l'Europe chrétienne son unité sous l'égide de l'Empire : «[...] tu pourras facilement conclure la paix dans la Chrétienté et étendre les frontières de l'Empire ${ }^{23}$." L'idée de cette souveraineté réapparaîtra dans la carte anthropomorphique de Sebastian Münster (1588) identifiant l'Europe sous les traits d'une reine tenant l'orbe impérial ${ }^{24}$.

7 Dans les écrits contemporains et, surtout, postérieurs à la prise de Constantinople, de Piccolomini, on note un emploi de plus en plus fréquent du mot «Europe », se substituant à celui de "Chrétienté » ou l'englobant : "Nous avons perdu une grande partie de l'Europe ; Mehmet [Mahomet II, sultan] nous a réduits dans un coin ${ }^{25}$ ", écrit-il à l'été 1454. Peuvent lui faire écho Georges de Trébizonde, intitulant un discours adressé au pape Nicolas V en octobre 1452, Pro defendenda Europa et Hellesponti claustra 
("Pour la défense de l'Europe et du détroit d'Hellespont »), et le pape Calixte III, s'engageant à faire « en sorte d'expulser totalement [les Turcs] hors des frontières d'Europe ${ }^{26}$ ». Dans le De Europa, le titre englobe à lui seul la conception du territoire ainsi désigné, et les emplois, rares, du mot «Europe » dans le corps du texte se limitent à indiquer le continent: "[cette flotte] entreprit d'empêcher les Turcs de passer d'Asie en Europe $^{27}$ »; ou bien, l'auteur déclare avoir, dans sa description, " parcouru les confins extrêmes de l'Europe $\mathrm{e}^{28}$ ». La raison de cette référence désormais récurrente à l'Europe s'explique, à mon sens, par la conscience, chez Piccolomini, que l'Europe ne se définit pas seulement par son identitée ${ }^{9}$ religieuse chrétienne, mais aussi par son héritage culturel grec et romain. C'est ainsi que, dans sa lettre à Nicolas $\mathrm{V}$ déplorant la ruine de Constantinople, il écrit :

Que dirais-je des livres innombrables qui se trouvaient en ce lieu, encore inconnus aux Latins? Las, combien de noms de grands hommes périront à présent? C'est la seconde mort d'Homère, le second trépas de Platon. Où rechercherons-nous les œuvres de génie des philosophes et des poètes ? [...] En Europe, sur notre sol, nous avons laissé l'ennemi s'emparer de la plus puissante ville de la Chrétienté, la capitale de l'Empire d'Orient, la colonne de la Grèce, le domicile des lettres ${ }^{30}$.

Sur la conscience de cet héritage qui fonde, aux yeux de Piccolomini, l'intégrité et l'unité de l'Europe, citons le propos de A. Ginzo Fernández : « Constantinople fait partie de la patrie, de la propre maison qu'est l'Europe. Aucun autre auteur médiéval n'avait pris une conscience aussi claire de cette pertinence, non plus que de l'européanité en tant que telle $e^{31}$.»

lorsque, devenu pape Pie II, Piccolomini écrira sa Lettre à Mahomet II, il opposera la décadence de la culture en terre d'islam à un tableau de l'Europe où le libre développement de la philosophie et de la théologie va de pair avec le foisonnement des centres universitaires :

Mais chez nous s'épanouissent pleinement les études des arts libéraux. Il y a des lectures publiques de philosophie, on tient des leçons de théologie dans les écoles, on ne délaisse aucun type de science, on trouve dans plusieurs villes d'Italie d'illustres universités de lettres, et au-delà des Alpes, en Espagne, en France, en Allemagne, en Angleterre, il ne manque pas de collèges d'hommes éminents ${ }^{32}$.

\section{Quels Européens ?}

«Européens ». J. Le Goff emploie constamment le mot pour l'Europe médiévale, en un temps où ce mot est totalement absent. Les Grecs avaient un substantif «europáioi » pour désigner ces Européens que sont, selon Hérodote, les Grecs. L'Antiquité latine a deux adjectifs : «europaeus» et, en bas-latin, «europensis», par ailleurs peu utilisés. L'Histoire Auguste, datant du IV siècle, mentionne des "armées d'Europe » («exercitus Europenses »), pour désigner les contingents occidentaux ${ }^{33}$. Au Moyen Âge, seuls deux emplois dans une chronique de 754 mentionnent les «Europenses » comme les Francs et leurs alliés victorieux des Arabes en $732^{34}$. Il faut attendre le De Europa de Piccolomini pour voir activer un emploi inédit d'« europaeus» comme substantif, là où il exprime initialement son intention de « transmettre brièvement à la postérité les faits, qu'on dit être dignes de mémoire et dont j'ai eu connaissance, survenus sous Frédéric, empereur troisième du nom, parmi les Européens et les insulaires qu'on désigne sous le nom de Chrétiens ${ }^{35} »$. 
11 La distinction qu'on pourrait percevoir entre « Européens » désignant les continentaux, et les insulaires, est inopérante dans le cours de l'ouvrage : tous ces «chrétiens » sont bien européens. L'appartenance à une communauté humaine pouvant s'identifier par des traits communs - la religion chrétienne - met en évidence la vision de Piccolomini, plus historique que géographique : il existe donc une identité collective distinguant des peuples aux origines diverses, dont les migrations d'abord, puis la sédentarisation ont configuré un paysage humain. L'emploi du terme «vultus» («visage ») est certes figuré, mais significatif : « tel est le visage de l'Europe, tel est l'état de la religion chrétienne ${ }^{36}$ ", écrit-il dans une lettre où il déplore les divisions qui altèrent la cause commune des peuples européens face à leur seul ennemi, le Turc, et de préciser: "Sans souci pour la chose publique, nous sommes esclaves de nos passions privées [...] Que nous procurerons-nous par la guerre civile ? [...] Mais les autres peuples d'Europe n'agissent pas mieux, il s'ensuivra pour chacun d'eux son propre désastre ${ }^{37}$. »

En termes politiques, les Européens constituent une même nation, attachés aux intérêts d'une même "chose publique » et leurs divisions sont comparées à une "guerre civile ». L'Europe de Piccolomini achève alors le mouvement de dilatation par l'intégration des populations baltes - Lituanie et Livonie christianisées depuis la fin du $\mathrm{XIV}^{\mathrm{e}}$ siècle seulement - compensant en partie la perte des peuples récemment tombés sous la domination des Turcs. Cependant, la dimension ethnique ne définit pas l'Europe :

Il existe encore, non loin de la source du Tanaïs, une deuxième Hongrie, souche de la nôtre [...] qui lui est presque semblable par la langue et les coutumes, bien que la nôtre soit plus civilisée [civilior], adorant le Christ ; celle-là vivant à la manière des barbares est l'esclave des idoles ${ }^{38}$.

Les composantes identitaires ( nostre », «nostra »), également fondées, on le constate, sur le rapport à l'altérité ( altera»), sont donc plus fortes que les facteurs de divisions, la communauté linguistique jouant un rôle secondaire de cohésion. Piccolomini, tout en étant sensible au caractère relatif de l'intégration de peuples naguère païens dans l'aire chrétienne et européenne n'en considère pas moins que l'élément intégrateur est à la fois d'ordre religieux (une foi commune), et d'ordre culturel, cette composante étant assez forte pour déterminer un degré de civilisation. La réactivation du clivage « civilisation » « barbarie » est un trait récurrent permettant de faire précisément des peuples qui forment l'Europe, des «Européens ». La Livonie est la dernière région balte acquise au christianisme et intégrée à l'Europe : «La religion chrétienne ouvrit cette partie de la terre à notre race, montrant à ses peuples particulièrement féroces, une fois purgée leur barbarie, un mode de vie plus doux ${ }^{39}$. " Ce n'est donc pas seulement le fait d'occuper une partie du territoire Europe qui fait l'appartenance à cette communauté. La région que Piccolomini appelle Romania (Roumanie) - en fait la Roumélie, c'est-à-dire toute la zone maritime jusqu'au détroit des Dardanelles qui était maintenant sous le contrôle des Turcs, correspondant à la Thrace et à la Macédoine est une parcelle d'Europe qui n'appartient plus à sa communauté, et il en mesure le recul saisissant vers le no man's land de la barbarie : " [Elle est] de nation grecque, bien qu'autrefois elle ait été barbare, et que, de nos jours, après la destruction de l'empire grec, sous la domination des Turcs, elle soit retournée à la barbarie ${ }^{40}$. »

La notion de mores (mœurs, style de vie) s'avère déterminante: Coluccio Salutati, en 1397, avertissait les chrétiens d'Europe de leur responsabilité dans la décadence des 
mœurs, raison pour lui beaucoup plus déterminante dans l'avancée des musulmans en Europe que la résistance armée ${ }^{41}$. Les éléments unificateurs d'une Europe des «Européens » existent donc pour Piccolomini en termes de culture autant que de religion.

\section{Un point de vue « italien » sur l'Europe ?}

Enea Silvio Piccolomini est un Siennois qui a accompli la moitié de sa carrière en Autriche, auprès de l'empereur Frédéric III de Habsbourg, dont il était devenu le secrétaire et le conseiller, à partir de 1442. Rappelons que la formation intellectuelle de Piccolomini s'est faite, et c'est là un fait déterminant, avant son départ pour le concile de Bâle en 1432, à 28 ans, par la fréquentation de maîtres humanistes au Studium de Sienne d'abord, puis à l'université de Florence auprès de Francesco Filelfo, puis à Padoue et à Ferrare où il rencontre l'humaniste Giovanni Aurispa. Lorsqu'il arrive à Vienne, il découvre d'abord la diversité linguistique et, parmi ses premières impressions, un peu à la manière des Persans de Montesquieu, il s'étonne que des Allemands puissent parler une langue qu'il ne comprend guère et qu'on ne puisse se faire comprendre des portiers en s'exprimant en latin : «Avec [le portier] je n'emploie pas le latin: je connais peu le teuton et, quand les mots me manquent, je me fais remplacer par un serviteur ${ }^{42}$." De même, il se demande comment ces Allemands peuvent apprécier une boisson aussi exécrable que la bière ${ }^{43}$. Il peut constater que les préjugés sont réciproques. Les Allemands se méfient des Italiens : «Le fait est que les Italiens sont trop rusés. Alors, à défaut d'autres moyens, ils utilisent le poison. » À quoi Enea répond : "Vous, les Allemands, [...] savez parfaitement simuler et dissimuler ${ }^{44}$.» Ces antipathies au XVI ${ }^{e}$ siècle ont fait l'objet de pages fort éclairantes par John Hale ${ }^{45}$. On rencontre, somme toute, peu de traits de ce type chez Piccolomini. Il est certes totalement convaincu de la supériorité et de l'avance de la culture des humanités - la sienne, «italienne »- sur une culture qu'il juge encore attachée aux vieilleries de la scolastique. En 1438, il en est encore à ses premières impressions alors qu'il découvre Vienne, et juge sévèrement le niveau de la culture universitaire de cette ville :

Du reste, ils n'ont d'intérêt ni pour la musique, ni pour la rhétorique ni pour l'arithmétique [...]. L'art oratoire et la poésie leur sont presque inconnus [...] On en trouve très peu qui possèdent des livres d'Aristote et d'autres philosophes, ils se servent généralement de commentaires. D'ailleurs, les étudiants eux-mêmes se donnent au plaisir, s'empiffrant de vin et de nourriture. Bien peu deviennent savants ${ }^{46}$.

16 Mais cette forme d'italo-centrisme est relative, car sa nouvelle situation à la cour de Vienne le mettra en contact avec des cercles amateurs de belles lettres latines. Les voyages du temps du concile lui avaient permis de satisfaire une curiosité pour les paysages et les contrées, curiosité jamais en défaut, qui le caractérise et s'exercera dans ses nombreux déplacements ultérieurs - Allemagne, Hongrie, Bohême. Il a découvert, par exemple, le style anglais, et dans ses Commentaires, écrits pendant son pontificat, il se souvient de l'impression très vive qu'il reçut de la cathédrale d'York ${ }^{47}$. À Vienne, il a admiré la verticalité et l'ampleur des intérieurs gothiques, et cette qualité propre de la lumière que leur confèrent les grandes verrières: «Des églises sont consacrées aux saints du Paradis et à Dieu souverain, vastes et splendides édifices, bâtis en pierre de taille, d'une lumineuse transparence, admirables par l'ordonnance de leurs colonnes $^{48}$.» 
L'église de Pienza devra essentiellement son architecture au modèle du gothique flamboyant des hallenkirchen (églises-halles) allemandes. Il fera de la richesse et la modernité de l'Allemagne une description remarquable dans son Germania ${ }^{49}$.

Sur l'ensemble des 65 chapitres qui, dans le De Europa, sont consacrés aux provinces, nations et peuples de l'Europe, dix-sept concernent l'Italie, le rapport étant favorable à la seule péninsule. Ces chapitres condensent la proportion la plus notable de références à la culture des lettres et des sciences. Ferrare se distingue par la protection donnée aux juristes et aux lettrés: "La maison des Este a toujours été amie des doctes ${ }^{50}$." Bologne est «la mère des études ${ }^{51}$ ", les Florentins se distinguent par "l'intérêt qu'ils portent à l'art oratoire et à ce qu'ils appellent les études d'humanités ${ }^{52}$ ». La Rome de Nicolas $\mathrm{V}$ doit à ce pape mécène, collectionneur de manuscrits, une exceptionnelle floraison artistique et culturelle : « En effet, Nicolas encouragea et favorisa les talents à tel point qu'on pourrait difficilement trouver une époque où les études d'humanité, d'éloquence et autres connaissances libérales aient davantage fleuri qu'à la sienne ${ }^{53}$.» Venise «a rendu illustre le nom italique chez les nations barbares ${ }^{54}$ ». Piccolomini exprime ainsi sa conscience d'une suprématie culturelle de la péninsule : lui-même, couronné poète par Frédéric III en juillet 1442, est persuadé d'exporter cette culture humaniste, dont le foyer reste l'Italie, dans des terres étrangères ${ }^{55}$.

La prise de Constantinople va tarir, selon lui, la source des lettres et de la philosophie, et dans ce chant funèbre, il s'inquiète de l'héritage classique qu'il appartient désormais à l'Europe de préserver et de faire fleurir, comme il l'écrit à Nicolas V :

Il restera quelque lumière chez les Latins, mais j'avoue que cela ne durera pas longtemps, à moins que Dieu n'abaisse vers nous un regard plus indulgent, et qu'il n'offre une fortune plus favorable à l'Empire romain et au Siège apostolique. Les lettres vivent avec le Siège romain, et sans lui toute science périt ${ }^{56}$.

L'héritage gréco-latin doit sa perpétuation aux deux grandes forces de cohésion: Empire et Papauté. Peu après la chute de l'Empire byzantin, Piccolomini rappelle que la source de la culture en Europe est la Grèce, et il en profite pour évoquer son ruissellement dans les principales villes universitaires du continent et d'Angleterre :

Le fleuve de toutes les doctrines est coupé, la source des Muses [allusion à Hippocrène] est tarie. Où rechercherons-nous maintenant la poésie, où la philosophie? Je reconnais qu'il est beaucoup d'endroits chez les Latins où brillent les études des lettres comme à Rome, Paris, Bologne, Padoue, Sienne, Pérouse, Cologne, Vienne, Salamanque, Oxford, Pavie, Erfurt. Mais ce sont tous là des ruisseaux, dérivés des sources des Grecs ${ }^{57}$.

Une majorité de villes italiennes sont citées, mais aussi de France, d'Espagne, d'Angleterre et d'Allemagne. Il est à ce propos remarquable que son sentiment d'appartenance à une "patrie » s'exprime sur trois modes : la "patrie» siennoise, la «patrie » italienne, la patrie européenne. Dans le De Europa, au moment de passer à la description des États italiens, il écrit qu'après avoir achevé de parcourir le nord de l'Europe, il est revenu « dans sa patrie ${ }^{58}$ ». J'ai déjà cité le « en Europe, sur notre sol » de la lettre du 12 juillet 1454 à Nicolas V. Enfin, s'adressant à Ratisbonne, en avril 1454, aux princes chrétiens d'Europe, il leur déclarait:

Il y a plusieurs siècles que la communauté chrétienne n'a souffert une plus grande ignominie. Car, dans les temps reculés, nous avons été frappés en Asie et en Afrique, c'est-à-dire dans des terres étrangères : mais à présent nous sommes frappés et attaqués en Europe, c'est-à-dire dans notre patrie, dans notre propre maison, dans notre foi ${ }^{59}$. 


\section{BIBLIOGRAPHIE}

BALDI Barbara, Pio II e le trasformazioni dell'Europa cristiana (1457-1464), Milan, Edizioni Unicopli, 2006.

CAPDEPUY Vincent, « Grands espaces et territorialité : le regard d'un pape géographe sur l'Europe, Æneas Sylvius Piccolomini (Pie II) », Cahiers de recherches médiévales et humanistes, $\mathrm{n}^{\circ} 21,2011$, p. 119-135. Disponible en ligne sur <http://crm.revues.org/12431>.

CHABOD Federico, Storia dell'idea d'Europa, Bari, Laterza, 1961, traduction française : Histoire de l'idée d'Europe, trad. de Yves Hersant, dans Y. Hersant et F. Durand-Bogaert (éds), Europes, de l'Antiquité au XXe siècle. Anthologie critique et commentée, Paris, R. Laffont, coll. « Bouquins », 2000, p. 209-312. de Historia de la Filosofia, vol. 28, 2011. 
HAY Denys, Europe. The Emergence of an Idea, Edimbourg, University Press, 1957.

Piccolomini Enea Silvio, De Europa, A. van Heck (éd.), Città del Vaticano, Biblioteca Apostolica Vaticana, 2001.

Pio II e la cultura del suo tempo, L. Rotondi Secchi Tarugi (éd.), Milan, Guerini e associati, 1991.

Pio II Piccolomini: il Papa del Rinascimento a Siena, F. Nevola (éd.), Colle Val d'Elsa, Protagon, 2009.

STOLF Serge, « Espace géographique et espace culturel : le De Europa d'Enea Silvio Piccolomini », Camenae, $\mathrm{n}^{\circ} 14,2012$. Disponible en ligne sur <http://saprat.ephe.sorbonne.fr/media/[...]/ camenae-14-2.pdf>.

Stolf Serge, Les Lettres et la Tiare. E. S. Piccolomini, un humaniste au XV $v^{e}$ siècle, Paris, Classiques Garnier, coll. « Études et essais sur la Renaissance », 2012.

Storia d'Europa, vol. 3, Il Medioevo. Secoli V-XV, Turin, Einaudi, 1994.

WOLKAN Rudolf, Der Briefwechsel des Eneas Silvius Piccolomini, dans Fontes Rerum Austriacarum: Diplomataria et Acta, series II, vol. 68, Vienne, 1918.

\section{NOTES}

1. F. Chabod, Storia dell'idea d'Europa, Bari, Laterza, 1961. Deux traductions françaises de ce livre sont à signaler, sous le titre Histoire de l'idée d'Europe : celle d'Yves Hersant, dans Y. Hersant et F. Durand-Bogaert, Europes, de l'Antiquité au $\mathrm{XX}^{e}$ siècle. Anthologie critique et commentée, Paris, R. Laffont, coll. «Bouquins », 2000, p. 209-312 (p. 210, pour la citation) ; celle de P.-L. van Berg, Éditions de l'Université de Bruxelles, 2014. Le livre de D. Hay, Europe. The Emergence of an Idea, Edimbourg, University Press, 1957, demeure une référence.

2. Poliziano, Stanze. Orfeo. Rime, D. Puccini (éd.), Milan, Garzanti, coll. « I grandi libri », 1995, p. 91 (1. I, CVI, v. 7).

3. Pour une étude générale sur le De Europa, on pourra consulter: B. Baldi, "Enea Silvio Piccolomini e il De Europa : umanesimo, religione e politica ", Archivio Storico Italiano, vol. 161, $\mathrm{n}^{\circ}$ 598, 2003, p. 619-683 ; Æneas Silvius Piccolomini, Europe (c. 1400-1458), Trans. R. Brown, Intr. and Notes N. Bisaha, Washington, Catholic University of America Press, 2013. Je me permets de renvoyer, pour des vues d'ensemble, à mon ouvrage : S. Stolf, Les Lettres et la Tiare. E. S. Piccolomini, un humaniste au $\mathrm{XV}^{e}$ siècle, Paris, Classiques Garnier, coll. "Études et essais sur la Renaissance ", 2012 ; pour une étude plus précise, voir Id., «Espace géographique et espace culturel: le De Europa d'Enea Silvio Piccolomini », Camenae, $\mathrm{n}^{\circ} 14,2012$, disponible en ligne sur <http:// saprat.ephe.sorbonne.fr/media/[...]/camenae-14-2.pdf>.

4. Sur cette réalité du territoire, voir, entre autres ouvrages: L. Provero, L'Italia dei poteri locali, Rome, Carocci, 1998.

5. Voir C. Vivanti, « Pio II e la cultura geografica del suo tempo », dans S. Gensini (éd.), Europa e Mediterraneo tra Medioevo e prima età moderna: l'osservatorio italiano, Pise, Pacini, 1992, p. 125-140.

6. Voir F. Guida, « Enea Silvio Piccolomini e l'Europa orientale : il "De Europa" (1458) », Clio, XV, 1979, p. 35-77.

7. Signalons, parmi les premières éditions du De Europa: In Europam, Memmingen, Albrecht Kunne, [antérieur à mars 1491] ; Europa, dans Cosmographia Pape Pij, Venetiis, per Bernardinum Venetum de Vitalibus (Bernardino dei Vitali), 1503. Sur ces premières éditions, voir L. Guerrini, «Umanesimo e geografia in E.S. Piccolomini», dans Nymphilexis. Enea Silvio Piccolomini, l'Umanesimo e la geografia, Rome, Edizioni Shakespeare and Company 2, 2005, p. 238-247. Je tends à penser qu'il s'agit d'une œuvre de circonstance, tout comme le Asia, écrit trois ans plus tard, et 
inachevé. Rien ne laisse croire, par ailleurs, que le De Europa ait été pensé comme première partie d'un diptyque (Europe et Asie), voire d'un triptyque (Afrique), jamais rédigé. Les deux ouvrages furent, en effet, publiés à Paris, en 1509, sous le titre Cosmographia Pii Papce in Asice et Europce eleganti descriptione, titre que conserveront les éditions successives du $\mathrm{XvI}^{\mathrm{e}}$ siècle.

8. J. Le Goff, L'Europe est-elle née au Moyen Âge ?, Paris, Éditions du Seuil, 2003, p. 11.

9. R. Fubini, «Enea Silvio Piccolomini nei suoi rapporti con la cultura umanistica del suo tempo », dans F. Nevola (éd.), Pio II Piccolomini: il Papa del Rinascimento a Siena, Colle Val d'Elsa, Protagon, 2009, p. 131-150. L'article fait le point sur la connaissance que Piccolomini avait des humanistes de sa génération (Alberti, Valla), et de ceux de la génération précédente, dont les noms reviennent souvent dans sa correspondance et dans son œuvre (Poggio, Bruni, Aurispa, Guarino Veronese).

10. S. Ratti, «L'Europe est-elle née dans l'Antiquité ? ", Anabases, $n^{\circ} 1,2005$, disponible en ligne sur <http://anabases.revues.org/1461>.

11. La traduction latine de Strabon fut achevée par G. Veronese en 1458. Voir N. Casella, «Pio II tra geografia e storia: la Cosmografia », Archivio della Società romana di Storia patria, vol. XCV, Rome, 1972, p. 70-71 ; L. Guerrini, « Geografia e politica in Pio II », dans Nymphilexis, ouvr. cité, p. 27-51.

12. E.S. Piccolomini, De Europa, A. van Heck (éd.), Città del Vaticano, Biblioteca Apostolica Vaticana, 2001, p. 71 (§ 29) : «tantum [est] spacium, quod Europam Asiamque disterminat; Bosphorum Thracium dixere maiores $"$.

13. Sur le "processus de territorialisation de l'Europe ", voir V. Capdepuy, "Grands espaces et territorialité : le regard d'un pape géographe sur l'Europe, Æneas Sylvius Piccolomini (Pie II)», Cahiers de recherches médiévales et humanistes, $\mathrm{n}^{\circ} 21,2011$, p.119-135, disponible en ligne sur $<$ http://crm.revues.org/12431>.

14. Cité par J. B. Bury, History of the Eastern Empire, from the Fall of Irene to the Accession of Basil: A.D. 802-867, New York, Cosimo Classics, 2008 [1912], p. 318.

15. J. Le Goff, L'Europe..., ouvr. cité, p. 50.

16. A. Paravicini Bagliani, «Il papato medievale e il concetto di Europa », dans Storia d'Europa, vol. 3, Il Medioevo. Secoli V-XV, Turin, Einaudi, 1994, p. 819-845 : «Dai testi carolingi emerge dunque un'Europa anzitutto germanica» (p. 822).

17. Ibid., p. 824, n. 22 : « a finibus Europae».

18. Ibid., p. 828, n. 42 : « Hanc igitur nostri mundi portiunculam Turci et Sarraceni bello premunt. »

19. E. S. Piccolomini, Epistolarium seculare, A. van Heck (éd.), Città del Vaticano, Biblioteca Apostolica Vaticana, 2007, p. 194: «[...] illa iam in angulo est christiani orbis. » (Lettre à Juan Carvajal, 23 octobre 1443)

20. Storia d'Europa, ouvr. cité, p. 832-833.

21. E. S. Piccolomini, Epistolarium, ouvr. cité, p. 87.

22. Oratio habita Basilece pro loco eligendo ad œecumenicum concilium cum Grecis celebrandum, dans Pii II P. M. olim Ænece Syluii Piccolominei Orationes politicce et ecclesiastica, J. D. Mansi (éd.), Lucæ, 1755-1759, pars I, p. 11 : « [Turci] qui ex Asia in Europa imperium prorogarunt [...], quos Grocia pellere non unius civitatis aut dominii, sed totius esset Christianitatis opus. »

23. E. S. Piccolomini, Epistolarium, ouvr. cité, p. 77 : «[...] facile poteris et pacem in christianitate componere et fines imperii propagare. » (Lettre à l'empereur Sigismond, 22 mai 1437)

24. Une reproduction se trouve dans: J. Hale, La civilisation de l'Europe à la Renaissance, Paris, Perrin, coll. « Tempus », 2003 [1993], p. 11.

25. R. Wolkan, Der Briefwechsel des Eneas Silvius Piccolomini, dans Fontes Rerum Austriacarum: Diplomataria et Acta, series II, vol. 68, Vienne, 1918, p. 544 : «Europe maximam partem amisimus; in angulum nos Maumethus coartavit. » (Lettre à Johann Vitéz, chancelier du royaume de Hongrie)

26. N. Iorga, Notes et extraits pour servir à l'Histoire des Croisades au XIVe siècle, série IV (1453-1476), Bucarest, Éd. de l'Académie roumaine, 1915, p. 118: «ut [...] a finibus Europe penitus expellantur » (lettre au roi Ladislas de Hongrie, 14 avril 1455). 
27. E. S. Piccolomini, De Europa, ouvr. cité, p. $69(\S 28)$ : «Turcis ex Asia transitum prohibere in Europam conata est. "

28. Ibid., p. 190 (§ 174) : «Ultimis Europe finibus peragratis [...] ».

29. Sur cette question de l'identité, voir C. Villain-Gandossi, «Identités et altérités de l'Europe jusqu'au Xve siècle », Hermès, La Revue, $n^{\circ}$ 23-24, 1999, p. 185-194.

30. R. Wolkan, Der Briefwechsel, ouvr. cité, p. 200 ; p. 201 : «quid de libris dicam, qui illic erant innumerabiles, nondum Latinis cogniti? heu, quot nunc magnorum nomina virorum peribunt? secunda mors ista Homero est, secundus Platoni obitus. ubi nunc philosophorum aut poetarum ingenia requiremus? [...] nos in Europa in nostro solo, inter Christianos potentissimam urbem, orientalis imperii caput, Grecie columen, litterarum domicilium ab hostili manu sinimus expugnari. "

31. A. Ginzo Fernández, «Eneas Silvio (Pío II) y su concepción de Europa », Anales del Seminario de Historia de la Filosofia, vol. 28, 2011, p. 88 : «Constantinopla forma parte de la patria, de la propia casa que es Europa. Ningún otro autor medieval había tomado una conciencia tan clara de esa pertinencia, ni tampoco de la europeidad como tal. »

32. Epistola ad Mahumetem, dans L. D'Ascia, Il Corano e la tiara. L'Epistola a Maometto II di Enea Silvio Piccolomini (papa Pio II), Bologne, Pendragon, 2001, p. 279 : «Inter nos vero liberalium artium studia admodum florent. Legitur publice philosophia, auditur in scholis theologia, nullum doctrince genus pretermittitur, gymnasia litterarum in pluribus Italice civitatibus clarissima reperiuntur, nec trans Alpes, in Hispania, in Gallia, in Germania, in Britannia collegia desunt virorum excellentium. » Sur cette lettre, qui a reçu une grande attention de la part des historiens, on peut consulter: Enea Silvio Piccolomini, Epistola al Gran Turco, A. Baldissera, A. Bresadola et G. Mazzocchi (éds), Côme, Ibis, 2008 ; J.-C. Margolin, «Place et fonction de la rhétorique dans la lettre de Pie II à Mahomet II ", dans L. Rotondi Secchi Tarugi (éd.), Pio II e la cultura del suo tempo, Milan, Guerini e associati, 1991, p. 243-261 ; N. Bisaha, « Pope Pius II's Letter to Sultan Mehmed II: A Reexamination », Crusades, vol. I, 2002, p. 183-200.

33. Histoire Auguste, édition bilingue, Paris, R. Laffont, coll. «Bouquins", Vie de Probus, p. 1088 : «Recepit deinde omnes Europenses exercitus» («Il plaça alors sous son commandement toutes les armées d'Europe », p. 1089).

34. P. Grattarola, « Il concetto di Europa alla fine del mondo antico », dans M. Sordi (éd.), L'Europa nel mondo antico, Contributi dell'Istituto di storia antica, vol.12, Milan, Pubblicazioni della Università Cattolica del Sacro Cuore, 1986, p. 174-191, cite des emplois du terme "Europenses » pour désigner les Francs et leurs alliés vainqueurs des Arabes en 732 (p. 190) : "diluculo prospiciunt Europenses Arabum temtoria» (Continuatio Hispana ad ann.754) (« au point du jour, les Européens observent les tentes des Arabes »).

35. E. S. Piccolomini, De Europa, ouvr. cité, p. 27 (prologue) : « Que sub Friderico, tertio eius nominis imperatore, apud Europeos et, qui nomine christiano censentur, insulares homines gesta feruntur memoratu digna mihique cognita, tradere posteris quam breuissime. " J. Hale, dans La civilisation (ouvr. cité, p. 3), affirme que l'on doit à Francis Bacon d'avoir forgé en 1623 l'expression « Nous, les Européens » (Nos Europäi). L'anticipation que constitue le De Europa ne fait pas de doute : D. Hay, dans Europe (ouvr. cité, p. 87), avait en effet souligné que « in Pius II, the word [Europaeus] has come to stay. Its usefulness made it have real significance ".

36. R. Wolkan, Der Briefwechsel, ouvr. cité, p. 283 : "Hic est Europe vultus, his status Christiane religionis » (lettre à Leonardo Benvoglienti, 25 septembre 1453).

37. Ibid., p. 279 et p. 282 : « rei publice nulla cura est, privatis affectibus inservimus. [...] Quid bello civili querimus? [...] Sed nec ceteri Europe populi melius agunt, sequetur sua quemque calamitas. »

38. E. S. Piccolomini, De Europa, ouvr. cité, p. 29 (§ 1) : « Extat adhuc non longe ab ortu Thanays altera Hungaria nostre huius, [...] mater, lingua et moribus pene similis, quamuis nostra ciuilior est, Christi cultrix; illa ritu barbarico uiuens seruit idolis. »

39. Ibid., p. 118-119 (§ 95) : « christiana religio hanc orbis partem nostro generi aperuit, que ferocissimis gentibus detersa barbarie mitioris uite cultum ostendit. » 
40. Ibid., p. 59 (\$ 18): " post quos [Bulgaros] loca maritima uersus meridiem ad Hellespontum usque Romania est, natio greca, quamuis olim barbara fuerit, et iterum nostro tempore deleto Grecorum imperio dominantibus Turcis in barbariam redit. »

41. Voir N. Husley, Crusading in the Fifteenth Century. Message and Impact, HoundmillsBasingstoke-Hampshire, Palgrave Macmillan, 2004, p. 16-17.

42. E. S. Piccolomini, Pentalogus, Herausgegeben von Christoph Schingnitz, Monumenta Germaniæ Historica, Hanovre, Hahnsche Buchhandlung, 2009, p. 58 : « Nec Latine ago secum. Pauca hec Teutonice scio et, cum deficio, puerum summitto. "

43. Piccolomini donne dans son De curialium miseriis un tableau, qui tient du documentaire et de la satire, de la vie de cour à Vienne : voir E. S. Piccolomini, Les Misères des gens de cour, suivi du Songe de Fortune, trad. et notes de S. Stolf, Grenoble, Ellug, 2015 : «Je ne parle pas de ces princes qui donnent à boire de la bière : cette boisson, amère partout, est néanmoins, servie à la cour, particulièrement amère et indigeste. » (p. 66)

44. E. S. Piccolomini, Pentalogus, ouvr. cité, p. 190 : « Nimium enim versipelles Itali sunt. Tum cum alia desunt, venenis utuntur »; "vos iam Germani [...] simulareque et dissimulare optime nostis ».

45. J. Hale, La civilisation, ouvr. cité, p. 53-69.

46. E. S. Piccolomini, Epistolarium, ouvr. cité, p. 79 : «ceterum neque musice neque rhetorice neque arismetrice curam gerunt [...]. oratorica et potica apud eos penitus incognita [...]. qui libros Aristotelis aliorumque philosophorum habeant raros inuenies, commentariis plerumque utuntur. ceterum studentes ipsi uoluptati operam prebent, uini cibique auidi. pauci emergunt docti. »

47. Voir Pii II Commentarii rerum memorabilium que temporibus suis contigerunt, A. van Heck (éd.), 2 vol., Città del Vaticano, Biblioteca Apostolica Vaticana, 1984, vol. 1, p. 48 (livre I, chap. 6).

48. E. S. Piccolomini, Epistolarium, ouvr. cité, p. 78 : «sanctis celum tenentibus ipsique maximo Deo templa dicata et ampla et splendida, secto lapide constructa, perlucida et columnarum ordinibus admiranda. " (Descriptio urbis Viennæe)

49. Æneas Silvius, Germania (und Jakob Wimpfeling: Responsa et replicee ad Eneam Silvium), herausgegeben von Adolf Schmidt, Bölhau, Köln, 1962.

50. E. S. Piccolomini, De Europa, ouvr. cité, p. 209 (§ 197-198) : «Fuit autem domus estensis uiris semper docis amica. "

51. Ibid., p. 220 (§ 199) : « studiorum mater».

52. Ibid. : « oratoriam spectant et que uocant humanitatis studia ».

53. Ibid., p. 243 (\$ 235) : « adeo enim ingenia excitauit fouitque Nicolaus, ut uix euum inueniri possit, in quo magis humanitatis et eloquentie ceterarumque bonarum artium studia quam suo floruerint. »

54. Ibid., p. 203 (§ 191) : « nomen italicum apud barbaras nationes [...] clarum [fecit] ».

55. Voir R. G. Witt, «Gli umanisti e l'Europa », dans G. Belloni et R. Drusi (éds), Il Rinascimento italiano e l'Europa, vol. II, Costabissara, Colla Editore, 2007, p. 97-114.

56. R. Wolkan, Der Briefwechsel, ouvr. cité, p. 211 : « restabit aliquid lucis apud Latinos, at fateor neque id erit diuturnum, nisi mitiori nos oculo Deus ex alto respexerit, fortunamque vel imperio Romano, vel apostolice sedi prebuerit meliorem. cum sede Romana vivunt littere, qua sublata perit omnis doctrina. »

57. Ibid., p. 210 : « precisus est fluvius doctrinarum, musarum dessicatus fons. ubi nunc poesis, ubi nunc philosophia requiremus? fateor multis locis apud Latinos studia litterarum esse illustria, ut Rome, Parisius, Bononie, Padue, Senis, Perusii, Colonie, Vienne, Salamantice, Oxonie, Papie, Erfordie. sed rivili sunt omnes isti, ex Grecorum fontibus derivati. »

58. E. S. Piccolomini, De Europa, ouvr. cité, p. 190 (§ 174) : «septentrione decurso in patriam tandem reversus".

59. Ænece Sylvii Piccolominei Senensis, qui post adeptum Pontificatum Pius eius nomini Secundus appelatus est, opera quce extant omnia..., Basileæ, per Henrichum Petri mense augusto an. MDLI, p. 678 : «multis ante seculis maiorem ignominiam passa est quam modo Christiana societas. Retroactis namque temporibus in Asia atque in Aphrica, hoc est in alienis terris uulnerati fuimus: nunc uero in 
Europa, id est in patria, in domo propria, in fide nostra percussi ccesique sumus. " (Oratio Ænece de Constantinopolitam Clade, et bello contra Turcos congregando)

60. E. S. Piccolomini, De Europa, ouvr. cité, p. 111 (§ 86) : « episcopus litterarum doctrina et morum suauitate insignis " ("évêque remarquable par sa science des lettres et par la douceur de ses mœurs »).

61. A. R. Baca, Selected letters of Æneas Silvius Piccolomini, San Fernando Valley State College, Northridge, CA, 1969, p. 115 : « conjungit epistola, quos terre mariaque disjungunt. nusquam tam procul amicus abiit, ut beneficio adiri non possit. 0 maximum ac divinum litterarum donum, quod non modo longe recedentes amicos, sed quos nunquam vidimus homines seu viventes sive mortuos in nostrum reducit alloquium et, quos carne intueri non possumus, mentibus offert conspiciendos. »

\section{RÉSUMÉS}

E. S. Piccolomini, bien avant de devenir pape en 1458 (Pie II), a orienté sa réflexion sur les problèmes politiques et culturels de son temps dans une perspective européenne. C'est tout l'intérêt de son ouvrage, De Europa, qu'il compose en 1458, où le passage de la Christianitas à l'Europa révèle la conception qu'un humaniste se fait, après la chute de l'Empire byzantin, des nouveaux rapports entre une identité européenne et un monde hostile à ses valeurs. Les «Européens » deviennent désormais une réalité définie par leur identité culturelle, humaine et spirituelle qu'il lui importe d'affirmer et de défendre.

E. S. Piccolomini, prima di diventare papa nel 1458 (Pio II), orientò la sua riflessione sui problemi politici e culturali del suo tempo in una prospettiva europea. In questo sta l'interesse del suo De Europa, scritto nel 1458, in cui il passaggio dalla Christianitas all'Europa rivela la concezione di un umanista, dopo la caduta dell'Impero bizantino, dei nuovi rapporti tra identità europea e un mondo ostile ai suoi valori. Gli «Europei» diventano ora una realtà definita dalla loro identità culturale, umana e spirituale che gli preme di affermare e di difendere.

E. S. Piccolomini, long before becoming pope in 1458 (Pius II), directed his reflection on the political and cultural problems of his time in a European perspective. This is the interest of his work, De Europa, which he composed in 1458, where the passage from Christianitas to Europa reveals the concept that a humanist is made after the fall of the Byzantine Empire, new relations between a European identity and a world hostile to its values. "Europeans" now become a reality defined by their cultural, human and spiritual identity that it is important for them to affirm and defend.

\section{INDEX}

Keywords : Piccolomini, Europe, Europeans, humanism, identity, Turks

Mots-clés : Piccolomini, Europe, Européens, humanisme, identité, Turcs

Parole chiave : Piccolomini, Europa, Europei, umanesimo, identità, Turchi 
AUTEUR

SERGE STOLF

LUHCIE, Université Grenoble Alpes

serge.stolf@wanadoo.fr 